\title{
The Linguistic Weighted Average
}

\author{
Dongrui Wu, Student Member, IEEE, and Jerry M. Mendel, Life Fellow, IEEE
}

\begin{abstract}
The focus of this paper is the linguistic weighted average (LWA), which is a generalization of the fuzzy weighted average (FWA) that is obtained by replacing the type-1 fuzzy inputs in the FWA by interval type-2 fuzzy sets (IT2 FSs). Consequently, the output of the LWA is an IT2 FS. In this paper, the relations between the LWA and the FWA are studied. It is shown that finding the LWA can be decomposed into finding two FWAs, where $\alpha$-cuts and KM algorithms are used. Hence, the computational cost of a LWA is about twice that of a FWA. A flowchart for computing the LWA is also provided.
\end{abstract}

\section{INTRODUCTION}

The weighted average (WA) is arguably the earliest and still most widely used form of aggregation. In this paper we focus on a new situation for the WA, one in which both the quantities being averaged (the attributes) as well as the weights are words. The resulting WA is called a linguistic WA (LWA). Our Example below illustrates a decision-making situation where the LWA is needed. First, however, we remind the reader of the well-known formula for the WA, i.e.

$$
y=\frac{\sum_{i=1}^{n} x_{i} w_{i}}{\sum_{i=1}^{n} w_{i}}=f\left(x_{1}, \ldots, x_{n}, w_{1}, \ldots, w_{n}\right)
$$

in which $w_{i}$ are the weights that act upon the attributes (e.g., decisions, features, indicators, etc.), $x_{i}$. Normalization is achieved by dividing the weighted numerator sum by the sum of all of the weights. While it is always true that the sum of the normalized weights that act upon each $x_{i}$ add to one, it is not a requirement that the sum of the un-normalized weights must add to one. In many situations requiring $\sum_{i=1}^{n} w_{i}=1$ is too restrictive; so, we do not impose such a requirement. It is the normalization that makes the calculation of the LWA very challenging.

In the LWA the weights are always words that are modeled as interval type- 2 fuzzy sets (IT2 FSs) [14], and the attributes may also be (but do not have to be) words that are also modeled as IT2 FSs ${ }^{1}$. We denote the LWA as $\tilde{Y}_{L W A}$, where

$$
\tilde{Y}_{L W A}=\frac{\sum_{i=1}^{n} \tilde{X}_{i} \tilde{W}_{i}}{\sum_{i=1}^{n} \tilde{W}_{i}}
$$

The tildes over all quantities denote IT2 FSs.

Before we formalize the LWA more carefully, it is instructive to provide an example that illustrates where it could be used.

Dongrui Wu and Jerry M. Mendel are with the Department of Electrical Engineering-Systems, University of Southern California, Los Angeles, CA 90089-2564, USA (phone: 213-740-4445; fax: 213-740-4651; email: dongruiw@usc.edu, mendel@ sipi.usc.edu).

${ }^{1}$ How to obtain IT2 FS models for words is an on-going research area, and one method for doing this can be found in [11] and [12]. In this paper, we assume that such models have already been established.
Example: Consider the following distributed and hierarchical decision-making situation. There are $n$ judges (or experts, managers, commanders, referees, etc.) who have to provide a subjective decision or judgement $\tilde{D}$ about a situation (e.g., quality of a submitted journal article). They will do this by providing a linguistic evaluation (i.e., a word, term, or phrase) for each of $m$ pre-specified and pre-ranked evaluation-categories, $C_{1}, C_{2}, \ldots, C_{m}$, using a pre-specified vocabulary of $t_{i}$ terms $(i=1,2, \ldots, m)$, because it may be too problematic to provide a numerical score for these categories. For a submitted journal article, the categories might be importance, content, depth, presentation, etc.; and, for e.g. presentation, the terms might be excellent, good, adequate, marginal and poor.

We assume that each of the category terms has been modeled a priori as an IT2 FS $\tilde{T}$; so, for each $C_{i}$ there is the associated IT2 FS $\tilde{T}_{C_{i}}$. Additionally, we assume that the $m$ evaluation-categories have also been linguistically rankordered a priori, so that each $C_{i}$ is associated with a linguistic weight, modeled as the IT2 FS $\tilde{W}_{i}$. The judges do not have to be concerned with any of the a priori rankings and modeling; it has all been done before they have been asked to judge.

After the judges have chosen a linguistic term for the $m$ categories, the following LWA is automatically computed:

$$
\tilde{D}_{j}=\frac{\sum_{i=1}^{m} \tilde{W}_{i} \tilde{T}_{C_{i}}}{\sum_{i=1}^{m} \tilde{W}_{i}} \quad j=1,2, \ldots, n .
$$

These $n$ IT2 FSs are then sent to a control (command) center (e.g., the associate editor); however, because judges may not be of equal expertise, we shall also assume that each judge's level-of-expertise has been pre-specified using a linguistic term $\tilde{T}_{J_{i}}$ provided by the judge from a small vocabulary of terms (e.g., low expertise, moderate expertise, high expertise). The linguistic evaluations from the $n$ judges, $\tilde{D}_{j}$, are then aggregated using a second LWA, as

$$
\tilde{D}=\frac{\sum_{j=1}^{n} \tilde{T}_{J_{j}} \tilde{D}_{j}}{\sum_{j=1}^{n} \tilde{T}_{J_{j}}}
$$

This second LWA is also sent to the control (command) center. Using $\tilde{D}_{j}(j=1,2, \ldots, n)$ and/or $\tilde{D}$, a final decision or judgement is made at the control (command) center.

There is a hierarchy of averages that can be associated with (1). We enumerate them next so that it will be clear where the LWA studied in this paper stands in this hierarchy.

1) $\forall w_{i}$ and $\forall x_{i}$ are crisp numbers: In this case, $y$ is a crisp number, the commonly-used arithmetic weighted average, a number that is easily computed using arithmetic. 
2) $\forall w_{i}$ are crisp numbers, and $\forall x_{i}$ are interval numbers, i.e. $x_{i}=\left[a_{i}, b_{i}\right]$ where interval end-points $a_{i}$ and $b_{i}$ are pre-specified: In this case, $y$ is an interval number (a weighted average of intervals), i.e. $y=\left[y_{l}, y_{r}\right]$, where $y_{l}$ and $y_{r}$ are easily computed [because interval sets only appear in the numerator of (1)] using interval arithmetic.

3) $\forall x_{i}$ are crisp numbers, and $\forall w_{i}$ are interval numbers, i.e. $w_{i}=\left[c_{i}, d_{i}\right]$ where interval end-points $c_{i}$ and $d_{i}$ are pre-specified: This is a special case of the fuzzy weighted average (FWA) [1], [8], [4], [7], [3], [2], [9] that also corresponds to the so-called centroid of an interval type-2 fuzzy set (IT2 FS) [14]. In this case, $y$ is also an interval number, i.e. $y=\left[y_{l}, y_{r}\right]$, but there are no known closed-form formulas for computing $y_{l}$ and $y_{r}$. The KM iterative algorithms [5], [14] have been used to compute $y_{l}$ and $y_{r}$. These algorithms are superexponentially and monotonically convergent [10], so it takes very few iterations for them to converge to the actual values of $y_{l}$ and $y_{r}$.

4) $\forall x_{i}$ are interval numbers, i.e. $x_{i}=\left[a_{i}, b_{i}\right]$ where interval end-points $a_{i}$ and $b_{i}$ are pre-specified, and $\forall w_{i}$ are interval numbers, i.e. $w_{i}=\left[c_{i}, d_{i}\right]$ where interval end-points $c_{i}$ and $d_{i}$ are pre-specified: This is another special case of the FWA that also corresponds to the so-called generalized centroid of IT2 FSs [14]. As in Case 3, $y$ is also an interval number, i.e. $y=$ $\left[y_{l}, y_{r}\right]$, but again there are no known closed-form formulas for computing $y_{l}$ and $y_{r}$. The KM iterative algorithms have also been used to compute $y_{l}$ and $y_{r}$.

5) $\forall x_{i}$ are type-1 fuzzy numbers, i.e. each $x_{i}$ is described by the membership function (MF) of a type-1 fuzzy set (T1 FS), $\mu_{X_{i}}\left(x_{i}\right)$, where this MF must be prespecified, and $\forall w_{i}$ are also type-1 fuzzy numbers, i.e. each $w_{i}$ is described by the MF of a T1 FS, $\mu_{W_{i}}\left(w_{i}\right)$, where this MF must also be pre-specified. This case is the FWA, and now $y$ is a T1 FS, with MF $\mu_{Y}(y)$, but there is no known closed-form formula for computing $\mu_{Y}(y)$. Recently, Liu and Mendel [9] showed how the $\mathrm{KM}$ algorithms can be used to compute an $\alpha$-cut decomposition [6] of $\mu_{Y}(y)$.

6) $\forall x_{i}$ are IT2 FSs, i.e. each $x_{i}$ is described by the footprint of uncertainty (FOU) of an IT2 FS, FOU $\left(\tilde{x}_{i}\right)$, where this FOU must be pre-specified, and $\forall w_{i}$ are also IT2 FSs, i.e. each $w_{i}$ is described by the FOU of an IT2 FS, FOU $\left(\tilde{w}_{i}\right)$, where this MF must also be pre-specified. Of course, there could be special subcases of this case, where only one or the other of the weights or attributes are IT2 FSs. This case is the LWA, and now $y$ is an IT2 FS.

In this work we focus on the LWA of Item 6 . The rest of this paper is organized as follows. Section II reviews the main results on the FWA, which serves as the basis to deduce the LWA algorithms. In Section III several theorems for the LWA are introduced. A flowchart for computing the LWA is presented in Section IV, followed by an example. Section V draws conclusions.

\section{The Fuzzy Weighted Average}

Because the idea of the FWA is used in the derivation of the LWA, it is briefly introduced in this section.

The FWA is defined as [1], [8], [4], [7], [3], [2], [9]:

$$
Y_{F W A}=\frac{\sum_{i=1}^{n} X_{i} W_{i}}{\sum_{i=1}^{n} W_{i}}
$$

Note that all $W_{i}$ and $X_{i}$ are T1 FSs. Consequently, $Y_{F W A}$ is also a T1 FS.

The FWA problem has been studied in multiple criteria decision making [1], [8], [4], [7], [3], [2] and computing the generalized centroid of an IT2 FS [5], [14], [13]. The fastest way to date to perform the computations are $\mathrm{KM}$ algorithms [9] introduced next.

In the KM algorithms approach, we first discretize the complete range of the membership $[0,1]$ of the fuzzy numbers $X_{1}, X_{2}, \ldots, X_{n}$ and $W_{1}, W_{2}, \ldots, W_{n}$ into $m \alpha$-cuts, $\alpha_{1}, \cdots, \alpha_{m}$. For each $\alpha_{j}$, we find the corresponding intervals for $x_{i}$ in $X_{i}$ and $w_{i}$ in $W_{i}(i=1,2, \ldots, n)$. Denote the endpoints of the intervals of $x_{i}$ and $w_{i}$ by $\left[a_{i}\left(\alpha_{j}\right), b_{i}\left(\alpha_{j}\right)\right]$ and $\left[c_{i}\left(\alpha_{j}\right), d_{i}\left(\alpha_{j}\right)\right]$, respectively, i.e.

$$
x_{i} \in\left[a_{i}\left(\alpha_{j}\right), b_{i}\left(\alpha_{j}\right)\right] \text { and } w_{i} \in\left[c_{i}\left(\alpha_{j}\right), d_{i}\left(\alpha_{j}\right)\right]
$$

The output of the FWA algorithm for this particular $\alpha$-cut, $Y_{F W A}\left(\alpha_{j}\right)$, is an interval, i.e.

$$
\begin{gathered}
Y_{F W A}\left(\alpha_{j}\right)=\frac{\sum_{i=1}^{n} X_{i}\left(\alpha_{j}\right) W_{i}\left(\alpha_{j}\right)}{\sum_{i=1}^{n} W_{i}\left(\alpha_{j}\right)} \\
=\left[\min _{\substack{\forall x_{i} \in\left[a_{i}\left(\alpha_{j}\right), b_{i}\left(\alpha_{j}\right)\right] \\
\forall w_{i} \in\left[c_{i}\left(\alpha_{j}\right), d_{i}\left(\alpha_{j}\right)\right]}} f\left(x_{1}, \ldots, x_{n}, w_{1}, \ldots, w_{n} \mid \alpha_{j}\right),\right. \\
\left.\max _{\substack{\forall x_{i} \in\left[a_{i}\left(\alpha_{j}\right), b_{i}\left(\alpha_{j}\right)\right] \\
\forall w_{i} \in\left[c_{i}\left(\alpha_{j}\right), d_{i}\left(\alpha_{j}\right)\right]}} f\left(x_{1}, \ldots, x_{n}, w_{1}, \ldots, w_{n} \mid \alpha_{j}\right)\right]
\end{gathered}
$$

where

$$
f\left(x_{1}, \ldots, x_{n}, w_{1}, \ldots, w_{n} \mid \alpha_{j}\right) \equiv \frac{\sum_{i=1}^{n} x_{i}\left(\alpha_{j}\right) w_{i}\left(\alpha_{j}\right)}{\sum_{i=1}^{n} w_{i}\left(\alpha_{j}\right)}
$$

It has been observed that [8], [5]

$$
\begin{aligned}
& \min _{\substack{\forall x_{i} \in\left[a_{i}\left(\alpha_{j}\right), b_{i}\left(\alpha_{j}\right)\right] \\
\forall w_{i} \in\left[c_{i}\left(\alpha_{j}\right), d_{i}\left(\alpha_{j}\right)\right]}} f\left(x_{1}, \ldots, x_{n}, w_{1}, \ldots, w_{n} \mid \alpha_{j}\right) \\
= & \min _{\substack{\forall w_{i} \in\left[c_{i}\left(\alpha_{j}\right), d_{i}\left(\alpha_{j}\right)\right] \\
\equiv}} \frac{\sum_{i=1}^{n} a_{i}\left(\alpha_{j}\right) w_{i}\left(\alpha_{j}\right)}{f_{L=1}^{n} w_{i}\left(\alpha_{j}\right)}
\end{aligned}
$$

and

$$
\begin{aligned}
& \max _{\substack{\forall x_{i} \in\left[a_{i}\left(\alpha_{j}\right), b_{i}\left(\alpha_{j}\right)\right] \\
\forall w_{i} \in\left[c_{i}\left(\alpha_{j}\right), d_{i}\left(\alpha_{j}\right)\right]}} f\left(x_{1}, \ldots, x_{n}, w_{1}, \ldots, w_{n} \mid \alpha_{j}\right) \\
= & \max _{\substack{\forall w_{i} \in\left[c_{i}\left(\alpha_{j}\right), d_{i}\left(\alpha_{j}\right)\right] \\
\equiv}} \frac{\sum_{i=1}^{n} b_{i}\left(\alpha_{j}\right) w_{i}\left(\alpha_{j}\right)}{f_{R}\left(\alpha_{j}\right)}
\end{aligned}
$$

These results are easy to prove because $X_{i}\left(\alpha_{j}\right)$ appear only in the numerator of (6), and so the smallest values of $X_{i}\left(\alpha_{j}\right)$ 


$$
\begin{aligned}
& f_{L}\left(\alpha_{j}\right)=\min _{\forall k \in[1, n-1]} \frac{\sum_{i=1}^{k} a_{i}\left(\alpha_{j}\right) d_{i}\left(\alpha_{j}\right)+\sum_{i=k+1}^{n} a_{i}\left(\alpha_{j}\right) c_{i}\left(\alpha_{j}\right)}{\sum_{i=1}^{k} d_{i}\left(\alpha_{j}\right)+\sum_{i=k+1}^{n} c_{i}\left(\alpha_{j}\right)} \equiv \frac{\sum_{i=1}^{k_{L}} a_{i}\left(\alpha_{j}\right) d_{i}\left(\alpha_{j}\right)+\sum_{i=k_{L}+1}^{n} a_{i}\left(\alpha_{j}\right) c_{i}\left(\alpha_{j}\right)}{\sum_{i=1}^{k_{L}} d_{i}\left(\alpha_{j}\right)+\sum_{i=k_{L}+1}^{n} c_{i}\left(\alpha_{j}\right)} \\
& f_{R}\left(\alpha_{j}\right)=\max _{\forall k \in[1, n-1]} \frac{\sum_{i=1}^{k} b_{i}\left(\alpha_{j}\right) c_{i}\left(\alpha_{j}\right)+\sum_{i=k+1}^{n} b_{i}\left(\alpha_{j}\right) d_{i}\left(\alpha_{j}\right)}{\sum_{i=1}^{k} c_{i}\left(\alpha_{j}\right)+\sum_{i=k+1}^{n} d_{i}\left(\alpha_{j}\right)} \equiv \frac{\sum_{i=1}^{k_{R}} b_{i}\left(\alpha_{j}\right) c_{i}\left(\alpha_{j}\right)+\sum_{i=k_{R}+1}^{n} b_{i}\left(\alpha_{j}\right) d_{i}\left(\alpha_{j}\right)}{\sum_{i=1}^{k_{R}} c_{i}\left(\alpha_{j}\right)+\sum_{i=k_{R}+1}^{n} d_{i}\left(\alpha_{j}\right)}
\end{aligned}
$$

are used to find the smallest value of (6), whereas the largest values of $X_{i}\left(\alpha_{j}\right)$ are used to find the largest value of (6).

Using KM algorithms [5], [9] presented in [15], $f_{L}\left(\alpha_{j}\right)$ and $f_{R}\left(\alpha_{j}\right)$ can be efficiently computed as (11) and (12) (given at the top of this page), where $k_{L}$ and $k_{R}$ are switch points satisfying

$$
\begin{aligned}
& a_{k_{L}}\left(\alpha_{j}\right) \leq f_{L}\left(\alpha_{j}\right) \leq a_{k_{L}+1}\left(\alpha_{j}\right) \\
& b_{k_{R}}\left(\alpha_{j}\right) \leq f_{R}\left(\alpha_{j}\right) \leq b_{k_{R}+1}\left(\alpha_{j}\right)
\end{aligned}
$$

When all $m$ intervals $\left[f_{L}\left(\alpha_{j}\right), f_{R}\left(\alpha_{j}\right)\right]$ are found, the MF of $Y_{F W A}, \mu_{Y_{F W A}}(y)$, is computed as

$$
\mu_{Y_{F W A}}(y)=\sup _{\forall \alpha_{j}(j=1, \ldots, m)} \alpha_{j} I_{Y_{F W A}\left(\alpha_{j}\right)}(y)
$$

where

$$
I_{Y_{F W A}\left(\alpha_{j}\right)}(y)= \begin{cases}1 & \forall y \in\left[f_{L}\left(\alpha_{j}\right), f_{R}\left(\alpha_{j}\right)\right] \\ 0 & \forall y \notin\left[f_{L}\left(\alpha_{j}\right), f_{R}\left(\alpha_{j}\right)\right]\end{cases}
$$

is an indicator function of $Y_{F W A}\left(\alpha_{j}\right)$.

\section{LWA THEORY}

The formulas for the LWA are derived in this section. For the convenience of the readers, we summarize all symbols used in the derivation in Table I. For notation simplicity and to save space, we omit the dependence on $\alpha_{j}$ in all symbols in the derivations. Readers should keep in mind that all the derivations are for a particular $\alpha$-cut, $\alpha_{j}$. Proofs of all theorems are in [15] and will be included in the journal version of this paper.

\section{A. Introduction}

The definition of the LWA is given in (2). Because an IT2 FS is completely determined by its FOU [14], $\tilde{Y}_{L W A}$ can also be expressed as

$$
\tilde{Y}_{L W A}=1 / F O U\left(\tilde{Y}_{L W A}\right) \equiv 1 /\left[\underline{Y}_{L W A}, \bar{Y}_{L W A}\right]
$$

where $\underline{Y}_{L W A}$ and $\bar{Y}_{L W A}$ are the lower and upper membership function (LMF and UMF) of $\tilde{Y}_{L W A}$, respectively, and the notation in (17) means that the secondary membership grade equals 1 at all elements in $F O U\left(\tilde{Y}_{L W A}\right)$. Hence, computing $\tilde{Y}_{L W A}$ is equivalent to computing $\underline{Y}_{L W A}$ and $\bar{Y}_{L W A}$.

\section{B. Computing the LWA}

$\alpha$-cuts are used to calculate $\underline{Y}_{L W A}$ and $\bar{Y}_{L W A}$. First, the complete range of the membership $[0,1]$ is discretized into $m \alpha$-cuts, $\alpha_{1}, \cdots, \alpha_{m}$; then, for each $\alpha_{j}$, the corresponding intervals for $x_{i}$ in $X_{i}$ and $w_{i}$ in $W_{i}$ are found, where $X_{i}$ and $W_{i}$ are embedded type- 1 fuzzy sets of $\tilde{X}_{i}$ and $\tilde{W}_{i}$ (see Fig. 1).
TABLE I

Notations USED IN THE DERIVATION. SEE ALSO Figs. 1 AND 5.

\begin{tabular}{c|c} 
Notation & Meaning \\
\hline$\tilde{X}_{i}$ & $i$ th attribute; an IT2 FS \\
$X_{i}$ & Embedded T1 FS of $\tilde{X}_{i}$ \\
$\bar{X}_{i}$ & UMF of $\tilde{X}_{i}$ \\
$\underline{X}_{i}$ & LMF of $\tilde{X}_{i}$ \\
{$\left[a_{i}\left(\alpha_{j}\right), b_{i}\left(\alpha_{j}\right)\right]$} & $\alpha$-cut on $X_{i}$ \\
{$\left[a_{i l}\left(\alpha_{j}\right), b_{i r}\left(\alpha_{j}\right)\right]$} & $\alpha$-cut on $\bar{X}_{i}$ \\
{$\left[a_{i r}\left(\alpha_{j}\right), b_{i l}\left(\alpha_{j}\right)\right]$} & $\alpha$-cut on $\underline{X}_{i}$ \\
\hline$\tilde{W}_{i}$ & Weight associated with $\tilde{X}_{i} ;$ an IT2 FS \\
$W_{i}$ & Embedded T1 FS of $\tilde{W}_{i}$ \\
$\bar{W}_{i}$ & UMF of $\tilde{W}_{i}$ \\
$\underline{W}_{i}$ & LMF of $\tilde{W}_{i}$ \\
{$\left[c_{i}\left(\alpha_{j}\right), d_{i}\left(\alpha_{j}\right)\right]$} & $\alpha$-cut on $W_{i}$ \\
{$\left[c_{i l}\left(\alpha_{j}\right), d_{i r}\left(\alpha_{j}\right)\right]$} & $\alpha$-cut on $\bar{W}_{i}$ \\
{$\left[c_{i r}\left(\alpha_{j}\right), d_{i l}\left(\alpha_{j}\right)\right]$} & $\alpha$-cut on $\underline{W}_{i}$ \\
\hline$\tilde{Y}_{L W A}$ & LWA computed from $\tilde{X}_{i}$ and $\tilde{W}_{i}$ \\
$\bar{Y}_{L W A}$ & UMF of $\tilde{Y}_{F W A}$ \\
$\underline{Y}_{L W A}$ & LMF of $\tilde{Y}_{F W A}$ \\
{$\left[f_{L}\left(\alpha_{j}\right), f_{R}\left(\alpha_{j}\right)\right]$} & $\alpha$-cut on an embedded T1 FS of $\tilde{Y}_{L W A}$ \\
{$\left[f_{L l}\left(\alpha_{j}\right), f_{R r}\left(\alpha_{j}\right)\right]$} & $\alpha$-cut on $\bar{Y}_{F W A}$ \\
{$\left[f_{L r}\left(\alpha_{j}\right), f_{R l}\left(\alpha_{j}\right)\right]$} & $\alpha$-cut on $\underline{Y}_{F W A}$ \\
\hline$h_{\max }$ & Maximum height of all $\underline{X}_{i}$ and all $\underline{W}_{i}$ \\
$h_{\min }$ & Minimum height of all $\underline{X}_{i}$ and all $\underline{W}_{i}$ \\
$U$ & See $(20)$ \\
$P_{\underline{X}}$ & See $(21)$ \\
$P_{W}$ & See $(22)$ \\
$a_{i r}^{\prime}\left(\alpha_{j}\right)$ & See $(45)$ \\
$b_{i l}^{\prime}\left(\alpha_{j}\right)$ & See $(46)$ \\
$c_{i r}^{\prime}\left(\alpha_{j}\right)$ & See $(49)$ \\
$d_{i l}^{\prime}\left(\alpha_{j}\right)$ & See $(50)$ \\
\hline &
\end{tabular}

We always use a normal IT2 FS; i.e. the maximum membership grades of the UMFs of all type-2 fuzzy sets equal unity. This means that each $\alpha$-cut on the UMFs will produce an interval for $\alpha_{j} \neq 1$, or at least, a crisp point for $\alpha_{j}=1$.

Generally, the LMFs of $\tilde{X}_{i}$ and $\tilde{W}_{i}$ have different heights (maximum membership grades), as shown in Figs. 2(a) and 2(b). Denote the height of $\underline{X}_{i}$ as $h_{\underline{X}_{i}}$, and the height of $\underline{W}_{i}$ as $h_{\underline{W}_{i}}$, respectively. Assume the maximum (minimum) height of all $\underline{X}_{i}$ and all $\underline{W}_{i}$ is $h_{\max }\left(h_{\min }\right)$, i.e.

$$
\begin{aligned}
& h_{\max }=\max \left\{\max _{\forall i \in[1, n]} h_{\underline{X}_{i}}, \max _{\forall i \in[1, n]} h_{\underline{W}_{i}}\right\} \\
& h_{\text {min }}=\min \left\{\min _{\forall i \in[1, n]} h_{\underline{X}_{i}}, \min _{\forall i \in[1, n]} h_{\underline{W}_{i}}\right\}
\end{aligned}
$$

Then, depending on the position of the $\alpha$-cut, there are three different cases:

1. $0 \leq \alpha_{j} \leq h_{\min }$ : the $\alpha$-cuts on all UMFs and LMFs exist, as shown in Fig. 2;

2. $h_{\min }<\alpha_{j} \leq h_{\max }$ : the $\alpha$-cuts on all UMFs exist while 


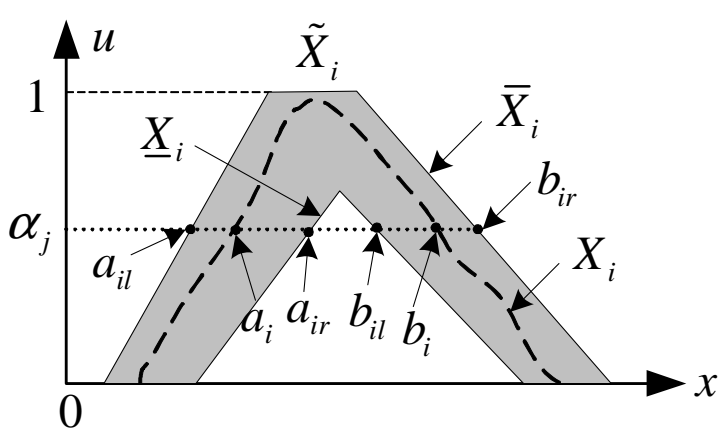

(a)

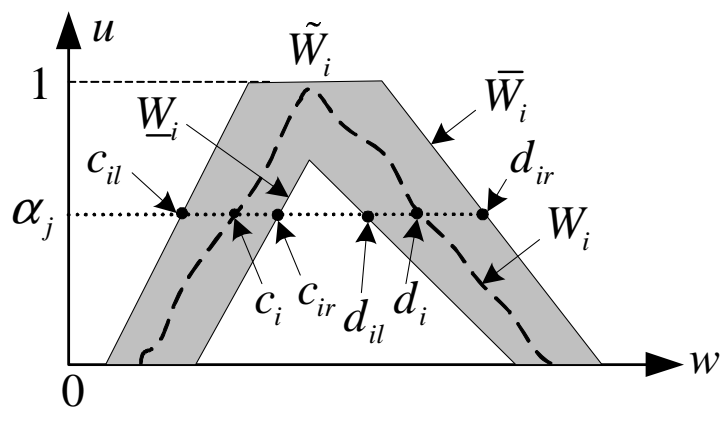

(b)

Fig. 1. The variables used in the derivation. (a) Variables for $\tilde{X}_{i}$; and, (b) Variables for $\tilde{W}_{i}$. The dashed curves are embedded T1 FSs.

the $\alpha$-cuts on some LMFs do not exist, as shown in Fig. 3;

3. $h_{\max }<\alpha_{j} \leq 1$ : the $\alpha$-cuts on all UMFs exist, but none of them exist on the LMFs, as shown in Fig. 4.

In order to distinguish between these three cases, we define

$$
U=\{1,2, \ldots, n\}
$$

and assume $P_{\underline{X}} \subseteq U$ and $P_{W} \subseteq U$ are finite sets consisting of integer indexes such that

$\left\{\begin{array}{l}\forall i \in P_{\underline{X}}, h_{\underline{X}_{i}}<\alpha_{j} ; a_{i r} \text { and } b_{i l} \text { do not exist } \\ \forall i \in U-\underline{P}_{\underline{X}}, h_{\underline{X}_{i}} \geq \alpha_{j} ; a_{i r} \text { and } b_{i l} \text { exist }\end{array}\right.$

and

$\left\{\begin{array}{l}\forall i \in P_{\underline{W}}, h_{\underline{\underline{W}}}<\alpha_{j} ; c_{i r} \text { and } d_{i l} \text { do not exist } \\ \forall i \in U-P_{\underline{\underline{W}}}, h_{\underline{W}_{i}} \geq \alpha_{j} ; c_{i r} \text { and } d_{i l} \text { exist }\end{array}\right.$

For example, in Fig. 2 we have $U=\{1,2,3\}, P_{\underline{X}}=\emptyset$ and $P_{\underline{W}}=\emptyset$; consequently, $\alpha_{j}$ in Fig. 2 produces intervals on all $\underline{X}_{i}$ and $\underline{W}_{i}$. In Fig. 3 we have $U=\{1,2,3\}, P_{X}=$ $\{1,2\}$ and $P_{\underline{W}}=\{3\}$; consequently, $\alpha_{j}$ in Fig. 3 does not produce intervals on $\underline{X}_{1}, \underline{X}_{2}$ and $\underline{W}_{3}$. In Fig. 4 we have $U=$ $\{1,2,3\}, P_{\underline{X}}=\{1,2,3\}$ and $P_{\underline{W}}=\{1,2,3\}$; consequently, $\alpha_{j}$ in Fig. $\overline{4}$ does not produce an interval on any $\underline{X}_{i}$ and $\underline{W}_{i}$.

We can now classify the three cases by using $P_{X}$ and $P_{W}$. When both $P_{\underline{X}}$ and $P_{\underline{W}}$ are empty, we are in Case 1; when both $P_{\underline{X}}$ and $P_{\underline{W}}$ equal $U$, we are in Case 3; otherwise, we are in Case 2 . Next, we shall consider the three cases individually.

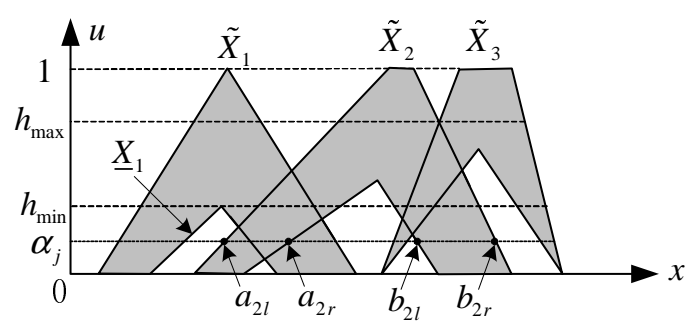

(a)

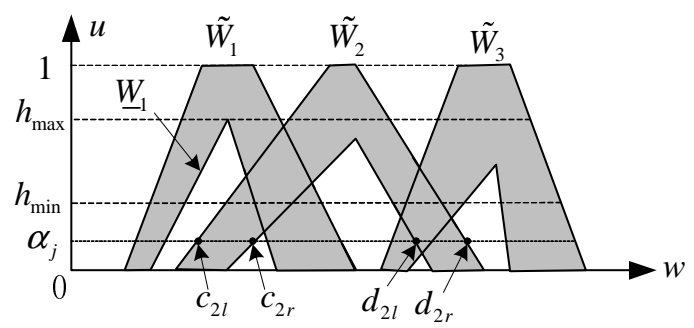

(b)

Fig. 2. Case 1: $0 \leq \alpha_{j} \leq h_{\text {min. }}$ Variables for (a) $\tilde{X}_{i}$ and (b) $\tilde{W}_{i}$.

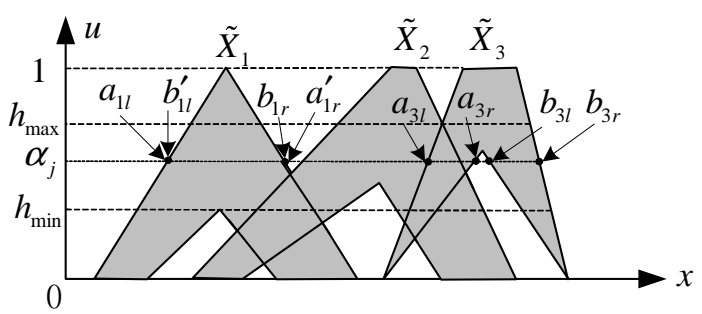

(a)

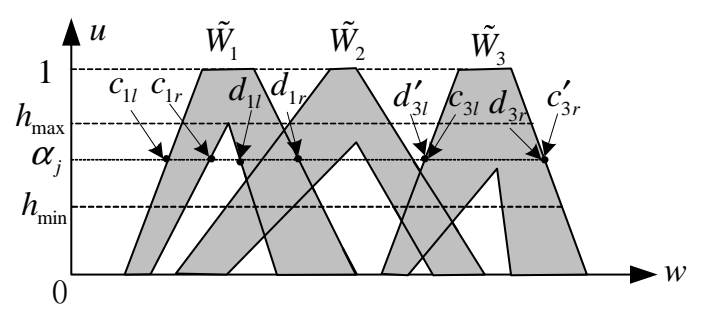

(b)

Fig. 3. Case 2: $h_{\min }<\alpha_{j} \leq h_{\max }$. Variables for (a) and (b) $\tilde{W}_{i}$.

C. Case 1: $0 \leq \alpha_{j} \leq h_{\min }$

When $0 \leq \alpha_{j} \leq h_{\text {min }}$, the $\alpha$-cuts on all UMFs and LMFs of $\tilde{X}_{i}$ and $\tilde{W}_{i}$ exist, as shown in Fig. 2. We denote the interval on $X_{i}$ as $\left[a_{i}, b_{i}\right]$, and the interval on $W_{i}$ as $\left[c_{i}, d_{i}\right]$, respectively. If we consider all the embedded $\mathrm{T} 1$ FSs, as shown in Figs. 1(a) and 1(b), then $a_{i} \in\left[a_{i l}, a_{i r}\right]$, $b_{i} \in\left[b_{i l}, b_{i r}\right], c_{i} \in\left[c_{i l}, c_{i r}\right]$ and $d_{i} \in\left[d_{i l}, d_{i r}\right]$.

Note that in (11) and (12) for the FWA, each of $a_{i}, b_{i}, c_{i}$ and $d_{i}$ can assume only one value; consequently, $f_{L}$ and $f_{R}$ are crisp numbers. However, in Case 1 of the LWA, $a_{i}, b_{i}, c_{i}$ and $d_{i}$ can assume values continuously in their corresponding $\alpha$-cut intervals. Numerous different combinations of $a_{i}, b_{i}$, $c_{i}$ and $d_{i}$ can be formed. $f_{L}$ and $f_{R}$ need to be computed for all the combinations. By collecting all $f_{L}$ we obtain a continuous interval $\left[f_{L l}, f_{L r}\right]$, and, by collecting all $f_{R}$ we 


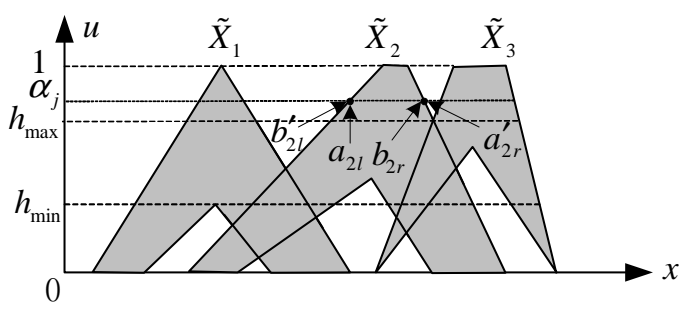

(a)

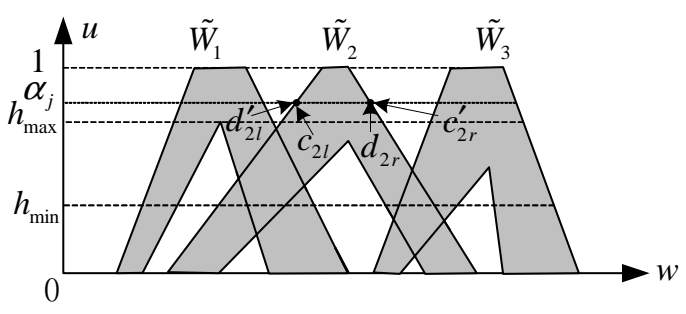

(b)

Fig. 4. Case 3: $h_{\max }<\alpha_{j} \leq 1$. Variables for (a) $\tilde{X}_{i}$ and (b) $\tilde{W}_{i}$.

obtain a continuous interval $\left[f_{R l}, f_{R r}\right]$, so that

$$
\underline{\mathrm{Y}}_{L W A}\left(\alpha_{j}\right)=\left[f_{L r}, f_{R l}\right]
$$

and

$$
\bar{Y}_{L W A}\left(\alpha_{j}\right)=\left[f_{L l}, f_{R r}\right]
$$

as shown in Fig. 5.

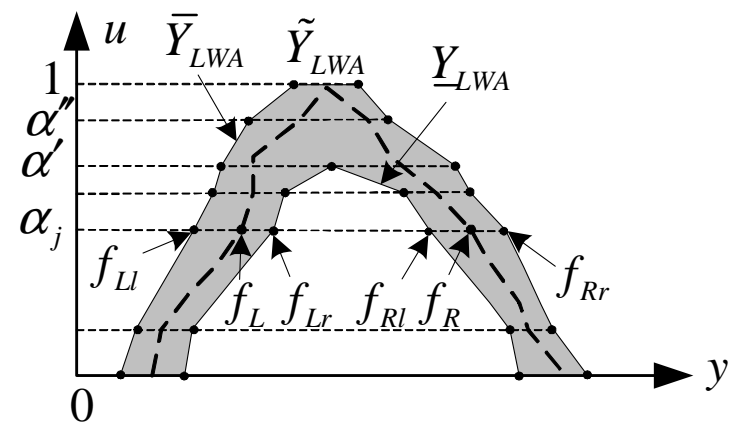

Fig. 5. Variables for $\tilde{Y}_{L W A}$.

To find $\underline{\mathrm{Y}}_{L W A}\left(\alpha_{j}\right)$ and $\bar{Y}_{L W A}\left(\alpha_{j}\right)$ we need to find $f_{L l}, f_{L r}, f_{R l}$ and $f_{R r}$. Consider $f_{L l}$ first. It is the minimum of $f_{L}$ [see (11)] when $a_{i} \in\left[a_{i l}, a_{i r}\right], c_{i} \in\left[c_{i l}, c_{i r}\right]$, and $d_{i} \in\left[d_{i l}, d_{i r}\right]$, i.e.

$$
f_{L l}=\min _{\substack{\forall a_{i} \in\left[a_{i l}, a_{i r}\right] \\ \forall c_{i} \in\left[c_{i l}, c_{i r}\right], \forall d_{i} \in\left[d_{i l}, d_{i r}\right]}} f_{L}
$$

Substituting $f_{L}$ in (11) into (25), we obtain

$$
\begin{aligned}
& f_{L l}=\min _{\substack{\forall a_{i} \in\left[a_{i l}, a_{i r}\right] \\
\forall c_{i} \in\left[c_{i j}, c_{i r}\right] \\
\forall d_{i} \in\left[d_{i l}, d_{i r}\right]}}\left[\min _{\forall k \in[1, n-1]} \frac{\sum_{i=1}^{k} a_{i} d_{i}+\sum_{i=k+1}^{n} a_{i} c_{i}}{\sum_{i=1}^{k} d_{i}+\sum_{i=k+1}^{n} c_{i}}\right] \\
& \equiv \min _{\substack{\forall a_{i} \in\left[a_{i l}, a_{i r}\right] \\
\forall c_{i} \in\left[c_{i i}, c_{i r}\right] \\
\forall d_{i} \in\left[d_{i l}, d_{i r}\right]}} \frac{\sum_{i=1}^{k_{L}} a_{i} d_{i}+\sum_{i=k_{L}+1}^{n} a_{i} c_{i}}{\sum_{i=1}^{k_{L}} d_{i}+\sum_{i=k_{L}+1}^{n} c_{i}}
\end{aligned}
$$

Because $a_{i}$ appear only in the numerator of (26), the smallest values of $a_{i}$ should be used to find the smallest value of (26). i.e.

$$
f_{L l}=\min _{\substack{\forall c_{i} \in\left[c_{i l}, c_{i r}\right] \\ \forall d_{i} \in\left[d_{i l}, d_{i r}\right]}} \frac{\sum_{i=1}^{k_{L 1}} a_{i l} d_{i}+\sum_{i=k_{L 1}+1}^{n} a_{i l} c_{i}}{\sum_{i=1}^{k_{L 1}} d_{i}+\sum_{i=k_{L 1}+1}^{n} c_{i}}
$$

where $k_{L 1}$ is the switch point for a particular combination of $\left(a_{1 l}, \ldots, a_{n l}, c_{i}, \ldots, c_{n}, d_{1}, \ldots, d_{n}\right)$.

Similarly, we can also express $f_{L r}, f_{R l}$ and $f_{R r}$ as

$$
\begin{gathered}
f_{L r}=\max _{\substack{\forall c_{i} \in\left[c_{i l}, c_{i r}\right] \\
\forall d_{i} \in\left[d_{i l} l, d_{i r}\right]}} \frac{\sum_{i=1}^{k_{L 2}} a_{i r} d_{i}+\sum_{i=k_{L 2}+1}^{n} a_{i r} c_{i}}{\sum_{i=1}^{k_{L 2}} d_{i}+\sum_{i=k_{L 2}+1}^{n} c_{i}} \\
f_{R l}=\min _{\substack{\forall c_{i} \in\left[c_{i l}, c_{i r}\right] \\
\forall d_{i} \in\left[d_{i l}, d_{i r}\right]}} \frac{\sum_{i=1}^{k_{R 1}} b_{i l} c_{i}+\sum_{i=k_{R 1}+1}^{n} b_{i l} d_{i}}{\sum_{i=1}^{k_{R 1}} c_{i}+\sum_{i=k_{R 1}+1}^{n} d_{i}} \\
f_{R r}=\max _{\substack{\left.\forall c_{i} \in c_{i l}, c_{i r}\right] \\
\forall d_{i} \in\left[d_{i l}, d_{i r}\right]}} \frac{\sum_{i=1}^{k_{R 2}} b_{i r} c_{i}+\sum_{i=k_{R 2}+1}^{n} b_{i r} d_{i}}{\sum_{i=1}^{k_{R} 2} c_{i}+\sum_{i=k_{R 2}+1}^{n} d_{i}}
\end{gathered}
$$

So far, we have only fixed $a_{i}$ for $f_{L l}$ and $f_{L r}$, and $b_{i}$ for $f_{R l}$ and $f_{R r}$. Next, we show that it is also possible to fix $c_{i}$ and $d_{i}$ for $f_{L l}, f_{L r}, f_{R l}$ and $f_{R r}$.

Theorem 1: It is true that

$$
a_{k_{L l}, l} \leq f_{L l} \leq a_{k_{L l}+1, l}
$$

and that $f_{L l}$ in (27) can be specified as

$$
f_{L l}=\frac{\sum_{i=1}^{k_{L l}} a_{i l} d_{i r}+\sum_{i=k_{L l}+1}^{n} a_{i l} c_{i l}}{\sum_{i=1}^{k_{L l}} d_{i r}+\sum_{i=k_{L l}+1}^{n} c_{i l}} ;
$$

where $k_{L l}$ is the switch point for $f_{L l}$. i.e., $f_{L l}$ is obtained by setting

$$
\left.\begin{array}{ll}
d_{i}=d_{i r} & \text { for } \quad i \leq k_{L l} \\
c_{i}=c_{i l} & \text { for } \quad i \geq k_{L l}+1
\end{array}\right\}
$$

in the right-hand side of (27). This means that $f_{L l}$ only depends on the UMF of $\tilde{W}_{i}, \bar{W}_{i}$.

Theorem 2: It is true that

$$
a_{k_{L r}, r} \leq f_{L r} \leq a_{k_{L r}+1, r}
$$

and that $f_{L r}$ in (28) can be specified as

$$
f_{L r}=\frac{\sum_{i=1}^{k_{L r}} a_{i r} d_{i l}+\sum_{i=k_{L r}+1}^{n} a_{i r} c_{i r}}{\sum_{i=1}^{k_{L r}} d_{i l}+\sum_{i=k_{L r}+1}^{n} c_{i r}} ;
$$

where $k_{L r}$ is the switch point for $f_{L r}$. i.e., $f_{L r}$ is obtained by setting

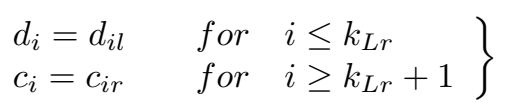

in the right-hand side of (28). This means that $f_{L r}$ only depends on the LMF of $\tilde{W}_{i}, \underline{W}_{i}$.

Theorem 3: It is true that

$$
b_{k_{R l}, l} \leq f_{R l} \leq b_{k_{R l}+1, l}
$$

and that $f_{R l}$ in (29) can be specified as

$$
f_{R l}=\frac{\sum_{i=1}^{k_{R l}} b_{i l} c_{i r}+\sum_{i=k_{R l}+1}^{n} b_{i l} d_{i l}}{\sum_{i=1}^{k_{R l}} c_{i r}+\sum_{i=k_{R l}+1}^{n} d_{i l}} ;
$$


where $k_{R l}$ is the switch point for $f_{R l}$. i.e., $f_{R l}$ is obtained by setting

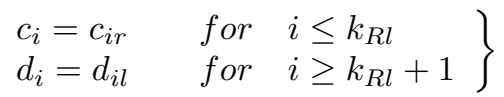

in the right-hand side of (29). This means that $f_{R l}$ only depends on the LMF of $\tilde{W}_{i}, \underline{W}_{i}$.

Theorem 4: It is true that

$$
b_{k_{R r}, r} \leq f_{R r} \leq b_{k_{R r}+1, r}
$$

and that $f_{R r}$ in (30) can be specified as

$$
f_{R r}=\frac{\sum_{i=1}^{k_{R r}} b_{i r} c_{i l}+\sum_{i=k_{R r}+1}^{n} b_{i r} d_{i r}}{\sum_{i=1}^{k_{R r}} c_{i l}+\sum_{i=k_{R r}+1}^{n} d_{i r}} ;
$$

where $k_{R r}$ is the switch point for $f_{R r}$. i.e., $f_{R r}$ is obtained by setting

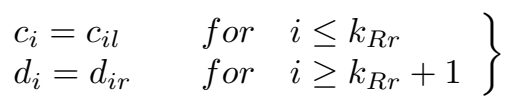

in the right-hand side of (30). This means that $f_{R r}$ only depends on the UMF of $\tilde{W}_{i}, \bar{W}_{i}$.

Using the above theorems we can show:

Theorem 5: $f_{L r} \leq f_{R l}$ for all $0 \leq \alpha_{j} \leq h_{\min }$. i.e., there is a gap $\left(f_{L r}, f_{R l}\right)$ between the left-hand interval $f_{L}=\left[f_{L l}, f_{L r}\right]$ and the right-hand interval $f_{R}=\left[f_{R l}, f_{R r}\right]$.

D. Case 2: $h_{\min }<\alpha_{j} \leq h_{\max }$

When $h_{\min }<\alpha_{j} \leq h_{\max }$, the $\alpha$-cuts on all UMFs exist. As shown in Section III-C, $f_{L l}$ and $f_{R r}$ depend only on the UMFs; thus, the formulas for them remain unchanged, i.e. Theorems 1 and 4 can still be used to compute $f_{L l}$ and $f_{R r}$ in Case 2. However, when $h_{\min }<\alpha_{j} \leq h_{\max }$, the $\alpha$-cuts on some LMFs do not exist; i.e. the $\alpha$-cut cannot produce intervals on those LMFs lower than $\alpha_{j}$, as shown in Figs. 3(a) and 3(b). Because $f_{L r}$ and $f_{R l}$ do depend on the LMFs, i.e., $f_{L r}$ in (35) depends on $a_{i r}, d_{i l}$ and $c_{i r}$, and $f_{R l}$ in (38) depends on $b_{i l}, c_{i r}$ and $d_{i l}$, we need to find new solutions for them in Case 2.

Comparing $a_{i}$ and $b_{i}$ with their ranges in Case 1, we see that they change in Case 2, i.e. (see Fig. 3)

$$
\begin{aligned}
& a_{i} \in\left[a_{i l}, a_{i r}^{\prime}\right] \\
& b_{i} \in\left[b_{i l}^{\prime}, b_{i r}\right]
\end{aligned}
$$

where

$$
a_{i r}^{\prime}= \begin{cases}b_{i r} & \forall i \in P_{\underline{X}} \\ a_{i r} & \forall i \in U-P_{\underline{X}}\end{cases}
$$

and

$$
b_{i l}^{\prime}= \begin{cases}a_{i l} & \forall i \in P_{\underline{X}} \\ b_{i l} & \forall i \in U-P_{\underline{X}}\end{cases}
$$

Similarly (see Fig. 3),

$$
\begin{aligned}
c_{i} & \in\left[c_{i l}, c_{i r}^{\prime}\right] \\
d_{i} & \in\left[d_{i l}^{\prime}, d_{i r}\right]
\end{aligned}
$$

where

$$
c_{i r}^{\prime}= \begin{cases}d_{i r} & \forall i \in P_{\underline{W}} \\ c_{i r} & \forall i \in U-P_{\underline{W}}\end{cases}
$$

and

$$
d_{i l}^{\prime}= \begin{cases}c_{i l} & \forall i \in P_{\underline{W}} \\ d_{i l} & \forall i \in U-P_{\underline{W}}\end{cases}
$$

Following the same procedure used to prove Theorem 2, we obtain:

Theorem $2^{\prime}:$ It is true that

$$
a_{k_{L r}, r}^{\prime} \leq f_{L r} \leq a_{k_{L r}+1, r}^{\prime}
$$

and that $f_{L r}$ in Case 2 can be specified as

$$
f_{L r}=\frac{\sum_{i=1}^{k_{L r}} a_{i r}^{\prime} d_{i l}^{\prime}+\sum_{i=k_{L r}+1}^{n} a_{i r}^{\prime} c_{i r}^{\prime}}{\sum_{i=1}^{k_{L r}} d_{i l}^{\prime}+\sum_{i=k_{L r}+1}^{n} c_{i r}^{\prime}} ;
$$

where $k_{L r}$ is the switch point for $f_{L r}$, and, $a_{i r}^{\prime}, c_{i r}^{\prime}$ and $d_{i l}^{\prime}$ are defined in (45), (49) and (50).

Theorem $3^{\prime}$ : It is true that

$$
b_{k_{R l}, l}^{\prime} \leq f_{R l} \leq b_{k_{R l}+1, l}^{\prime}
$$

and that $f_{R l}$ in Case 2 can be specified as

$$
f_{R l}=\frac{\sum_{i=1}^{k_{R l}} b_{i l}^{\prime} c_{i r}^{\prime}+\sum_{i=k_{R l}+1}^{n} b_{i l}^{\prime} d_{i l}^{\prime}}{\sum_{i=1}^{k_{R l}} c_{i r}^{\prime}+\sum_{i=k_{R l}+1}^{n} d_{i l}^{\prime}} ;
$$

where $k_{R l}$ is the switch point for $f_{R l}$, and, $b_{i l}^{\prime}, c_{i r}^{\prime}$ and $d_{i l}^{\prime}$ are defined in (46), (49) and (50).

Observe, from Fig. 5, that when $f_{L r}<f_{R l}$, the interval $\left[f_{L l}, f_{R r}\right]$ determined by the $\alpha$-cut on the UMFs is divided into two sub-intervals, $\left[f_{L l}, f_{L r}\right]$ and $\left[f_{R l}, f_{R r}\right]$, which are separated by a gap $\left(f_{L r}, f_{R l}\right)$. The FOUs must lie within $\left[f_{L l}, f_{L r}\right]$ and $\left[f_{R l}, f_{R r}\right]$, but they cannot enter the gap $\left(f_{L r}, f_{R l}\right)$. What if $f_{L r} \geq f_{R l}$ ? This cannot happen in Case 1 (see Theorem 5) but may happen in Case 2, because in Case 2 the ranges of $a_{i}, b_{i}, c_{i}$ and $d_{i}$ are extended, and the extended ranges have the effect of eliminating the gap. Observe that when $f_{L r} \geq f_{R l}$, the right bound of $\left[f_{L l}, f_{L r}\right]$ exceeds (or equals) the left bound of $\left[f_{R l}, f_{R r}\right]$. i.e. the two intervals $\left[f_{L l}, f_{L r}\right]$ and $\left[f_{R l}, f_{R r}\right]$ partially overlap and the gap is covered. When that happens, the FOU of $\tilde{Y}_{L W A}$ lies completely within the interval $\left[f_{L l}, f_{R r}\right]$, which is shown at $\alpha_{j}=\alpha^{\prime \prime}$ in Fig. 5 .

Although we stated Theorems $2^{\prime}$ and $3^{\prime}$ in the context of Case 2, they are not limited to Case 2. Actually, we used (43), (44), (47) and (48) instead of $h_{\min }<\alpha_{j} \leq h_{\max }$ in the derivations, and these equations can also represent Case 1 by properly setting their parameters, i.e., letting all $P_{\underline{W}}$ and $P_{\underline{X}}$ be empty sets. It is easy to show that (52) and (54) for Case 2 coincide with (35) and (38) in Case 1, respectively. This means that Theorems $2^{\prime}$ and $3^{\prime}$ can also be used to calculate $f_{L r}$ and $f_{R l}$ in Case 1, i.e., they give the same outputs as those of Theorems 1 and 2. In the next subsection we will show that Theorems $2^{\prime}$ and $3^{\prime}$ can also be applied to Case 3 .

\section{E. Case 3: $h_{\max }<\alpha_{j} \leq 1$}

When $h_{\max }<\alpha_{j} \leq 1$, the $\alpha$-cuts on all UMFs exist. Consequently, Theorems 1 and 4 can still be used to compute $f_{L l}$ and $f_{R r}$ in Case 3. However, none of the $\alpha$-cuts on the LMFs of $\tilde{X}_{i}$ and $\tilde{W}_{i}$ exists; thus, we need to find new solution for $f_{L r}$ and $f_{R l}$ in this case. 
Observe that Case 3 can also be represented by (43), (44), (47) and (48) by setting all $P_{X}$ and $P_{W}$ to $U$; thus, Theorems 6 and 7 in Section III-D can also be used here to compute $f_{L r}$ and $f_{R l}$. Because $P_{\underline{X}}$ and $P_{\underline{W}}$ are $U$, (45), (46), (49) and (50) in Section III-D become

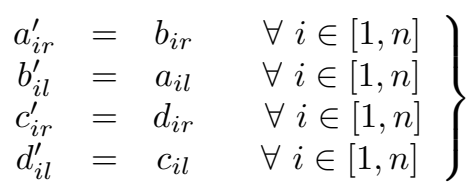

Substituting (55) into (52) and (54), we obtain

$$
\begin{aligned}
f_{L r} & =\frac{\sum_{i=1}^{k_{L r}} b_{i r} c_{i l}+\sum_{i=k_{L r}+1}^{n} b_{i r} d_{i r}}{\sum_{i=1}^{k_{L r}} c_{i l}+\sum_{i=k_{L r}+1}^{n} d_{i r}} \\
f_{R l} & =\frac{\sum_{i=1}^{k_{R l}} a_{i l} d_{i r}+\sum_{i=k_{R l}+1}^{n} a_{i l} c_{i l}}{\sum_{i=1}^{k_{R l}} d_{i r}+\sum_{i=k_{R l}+1}^{n} c_{i l}}
\end{aligned}
$$

Note that $f_{L l}$ and $f_{R r}$, which determine the $\alpha$-cut on $\bar{Y}_{L W A}$, are calculated by (32) and (41), respectively. Comparing (56) with (41), it is observed that $f_{L r}$ in (56) is the same as $f_{R r}$ in (41) ${ }^{2}$. Besides, $f_{R l}$ in (57) is also the same as $f_{L r}$ in (32); thus, in Case 3,

$$
\begin{aligned}
& f_{L l}=f_{R l} \\
& f_{L r}=f_{R r}
\end{aligned}
$$

Consequently,

$$
\left[f_{L l}, f_{L r}\right]=\left[f_{R l}, f_{R r}\right]=\left[f_{L l}, f_{R r}\right]
$$

(60) means that the FOU of $\tilde{Y}_{L W A}$ fills in the entire interval $\left[f_{L l}, f_{R r}\right]$ (see $\alpha^{\prime \prime}$ in Fig. 5), which is completely determined by the $\alpha$-cuts on the UMFs.

Theorem 6: When $h_{\max }<\alpha_{j} \leq 1$, the FOU of $\tilde{Y}_{L W A}$ fills in the entire interval $\left[f_{L l}, f_{R r}\right]$. Consequently, there is no need to calculate $f_{L r}$ and $f_{R l}$.

\section{F. Relations between the LWA and the FWA}

By summarizing the results above, we can connect the LWA and the FWA.

Theorem 7: The UMF of the LWA, $\bar{Y}_{L W A}$, is completely determined by the UMFs of the attributes, $\bar{X}_{i}$, and the UMFs of the corresponding weights, $\bar{W}_{i}(i=1,2, \ldots, n)$. More specifically, $\bar{Y}_{L W A}$ is the FWA of $\bar{X}_{i}$ and $\bar{W}_{i}$. i.e. let

$$
Y_{F W A}=\frac{\sum_{i=1}^{n} \bar{X}_{i} \bar{W}_{i}}{\sum_{i=1}^{n} \bar{W}_{i}}
$$

Then,

$$
\bar{Y}_{L W A}=Y_{F W A}
$$

Generally, it is impossible to find a similar theorem for $\underline{Y}_{L W A}$, because to compute $\underline{Y}_{L W A}$ we need to consider three different cases; however, we can do that for a special case of $\underline{Y}_{L W A}$, where all LMFs of $\tilde{X}_{i}$ and $\tilde{W}_{i}$ have the same height.

\footnotetext{
${ }^{2}$ The switch point in (56) is denoted as $k_{L r}$ and that in (41) is denoted as $k_{R r}$; however, because all $b_{i r}, c_{i l}$ and $d_{i r}$ are the same in (56) and (41), when the KM algorithm is used to compute (56) and (41), the resulting switch points will be the same. Consequently, (56) and (41) are the same.
}

Theorem 8: If all LMFs of $\tilde{X}_{i}$ and $\tilde{W}_{i}$ have the same height, then $\underline{Y}_{L W A}$ is the FWA of $\underline{X}_{i}$ and $\underline{W}_{i}$. i.e. let

$$
Y_{F W A}^{\prime}=\frac{\sum_{i=1}^{n} \underline{X}_{i} \underline{W}_{i}}{\sum_{i=1}^{n} \underline{W}_{i}}
$$

Then,

$$
\underline{Y}_{L W A}=Y_{F W A}^{\prime}
$$

\section{LWA ALgORITHMS}

A flowchart of the LWA algorithms is shown in Fig. 6. To save computational cost, different $\alpha$-cuts are chosen for $\underline{Y}_{L W A}$ and $\bar{Y}_{L W A}$ [15]. The procedures in the two dashed rectangles can be computed in parallel. Furthermore, the $\mathrm{KM}$ algorithms in the two dotted rectangles in each dashed rectangle can also be computed in parallel. The detailed algorithms are given in [15].

As an example, consider $\tilde{X}_{i}$ and $\tilde{W}_{i}$ shown in Fig. 7(a) and 7(b), respectively. The resulting $\tilde{Y}_{L W A}$ is shown in Fig. 7(c). $201 \alpha$-cuts were employed. The dashdot curve in Fig. 7(c) indicates the overlapped area where $f_{L r}\left(\alpha_{j}\right)>f_{R l}\left(\alpha_{j}\right)$ (see Section III-D).

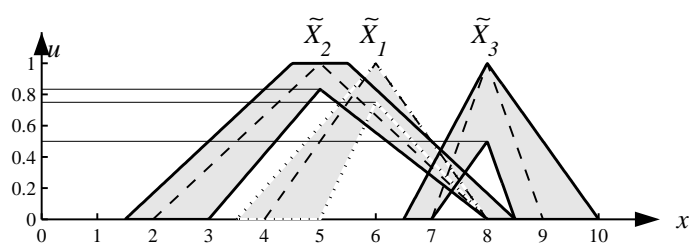

(a)

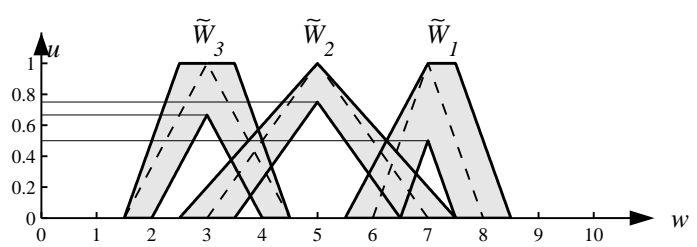

(b)

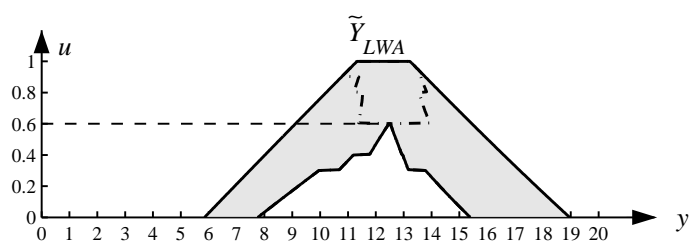

(c)

Fig. 7. MFs of (a) $\tilde{X}_{i}$ (b) $\tilde{W}_{i}$ and (c) $\tilde{Y}_{L W A}$.

\section{CONClusions}

In this paper, we have introduced the concept of the LWA. $\alpha$-cuts and KM algorithms were employed to compute it. Because the LWA is a generalization of the FWA from T1 FSs to IT2 FSs, there should be close relations between them. We have shown that finding the LWA, $\tilde{Y}_{L W A}$, is equivalent to finding its UMF, $\bar{Y}_{L W A}$, and LMF, $\underline{Y}_{L W A}$. Moreover, $\bar{Y}_{L W A}$ is the FWA of the UMFs of the attributes, $\tilde{X}_{i}$, and the UMFs of the corresponding weights, $\tilde{W}_{i} \cdot \underline{Y}_{L W A}$ is 


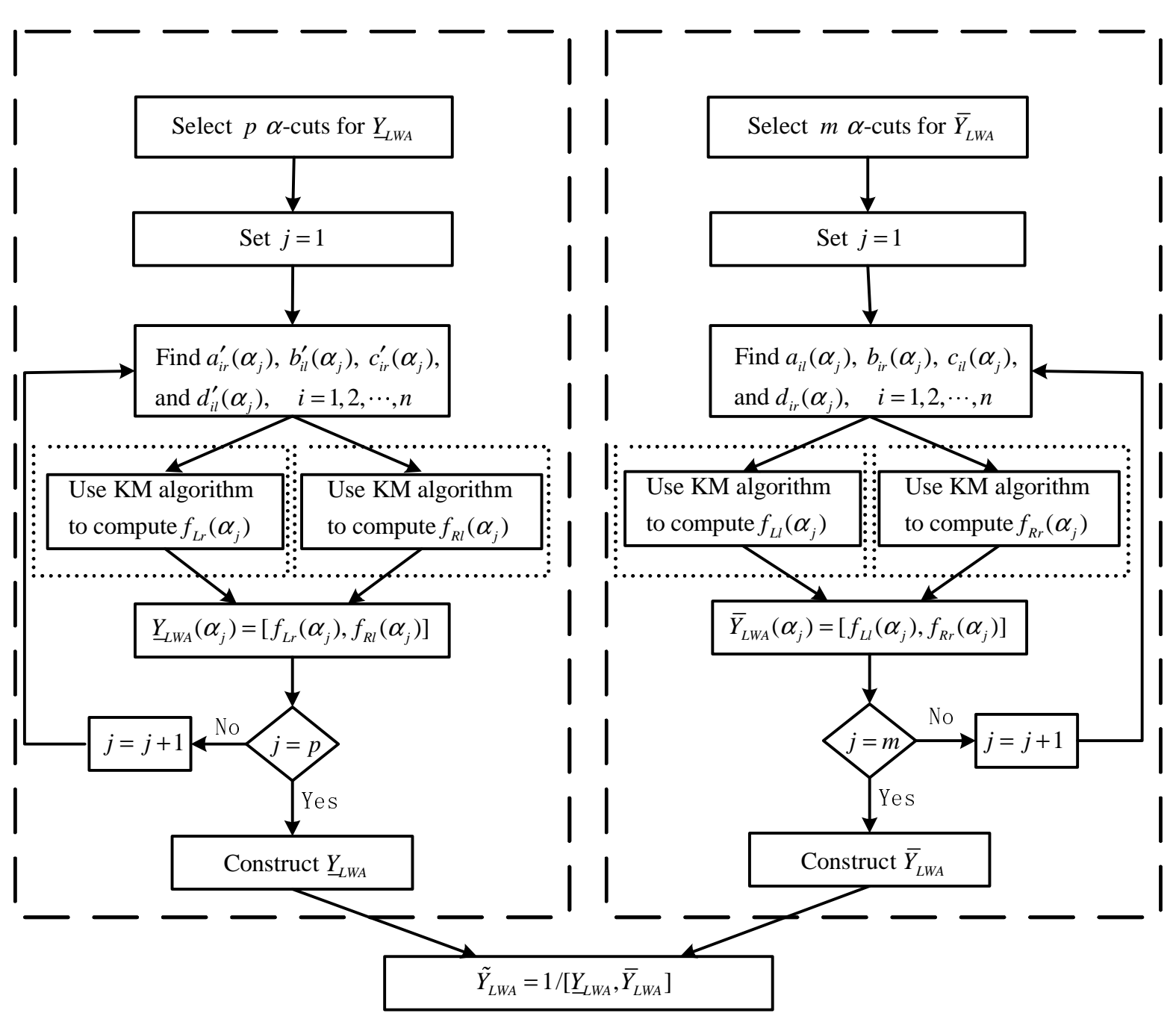

Fig. 6. Flowchart for the LWA.

more complicated than $\underline{Y}_{L W A}$, but it can also be computed efficiently by using $\alpha$-cuts and KM algorithms. For the special case where all the LMFs of $\tilde{X}_{i}$ and $\tilde{W}_{i}$ have the same height, $\underline{Y}_{L W A}$ is the FWA of the LMFs of $\tilde{X}_{i}$ and $\tilde{W}_{i}$. Hence, the computational cost of a LWA is about twice that of a FWA.

\section{ACKNOWLEDGEMENT}

This study was funded by the Center of Excellence for Research and Academic Training on Interactive Smart Oilfield Technologies (CiSoft); CiSoft is a joint University of Southern California-Chevron initiative.

\section{REFERENCES}

[1] W. M. Dong and F. S. Wong, "Fuzzy weighted averages and implementation of the extension priniple," Fuzzy sets and systems, vol. 21, pp. 183-199, 1987.

[2] D. Dubois, H. Fargier, and J. Fortin, "A generalized vertex method for computing with fuzzy intervals," in Proc. FUZZ-IEEE, Budapest, Hungary, July 2004, pp. 541-546.

[3] Y.-Y. Guh, C.-C. Hon, and E. S. Lee, "Fuzzy weighted average: The linear programming approach via Charnes and Cooper's rule," Fuzzy Sets and Systems, vol. 117, pp. 157-160, 2001.

[4] Y.-Y. Guh, C.-C. Hon, K.-M. Wang, and E. S. Lee, "Fuzzy weighted average: A max-min paired elimination method," J. of Computers and Mathematics with Application, vol. 32, pp. 115-123, 1996.
[5] N. N. Karnik and J. M. Mendel, "Centroid of a type-2 fuzzy set," Information Sciences, vol. 132, pp. 195-220, 2001.

[6] G. J. Klir and B. Yuan, Fuzzy Sets and Fuzzy Logic: Theory and Applications. Upper Saddle River, NJ: Prentice-Hall, 1995.

[7] D. H. Lee and D. Park, "An efficient algorithm for fuzzy weighted average," Fuzzy sets and systems, vol. 87, pp. 39-45, 1997.

[8] T.-S. Liou and M.-J. J. Wang, "Fuzzy weighted average: An improved algorithm," Fuzzy Sets and Systems, vol. 49, pp. 307-315, 1992.

[9] F. Liu and J. M. Mendel, "Aggregation using the fuzzy weighted average, as computed using the Karnik-Mendel algorithms," accepted for publication in IEEE Trans. on Fuzzy Systems, 2006.

[10] J. M. Mendel and F. Liu, "Super-exponential convergence of the Karnik-Mendel algorithms for computing the centroid of an interval type-2 fuzzy set," accepted for publication in IEEE Trans. on Fuzzy Systems, 2006.

[11] J. M. Mendel and H. Wu, "Type-2 fuzzistics for symmetric interval type-2 fuzzy sets: Part 1, forward problems," IEEE Trans. on Fuzzy Systems, 2006, in press.

[12] _ - "Type-2 fuzzistics for symmetric interval type-2 fuzzy sets: Part 2, inverse problems," IEEE Trans. on Fuzzy Systems, 2006, in press.

[13] _ - "New results about the centroid of an interval type-2 fuzzy set, including the centroid of a fuzzy granule," Information Sciences, vol. 177, pp. 360-377, 2007.

[14] J. M. Mendel, Rule-Based Fuzzy Logic Systems: Introduction and New Directions. Upper Saddle River, NJ: Prentice-Hall, 2001.

[15] D. Wu and J. M. Mendel, "On the linguistic weighted average," Signal and Image Processing Institute, University of Southern California, Los Angeles, CA, Tech. Rep., 2006. 